JOURNAL DE PHYSIQUE IV

Colloque C7, supplément au Journal de Physique III, Volume 4, juillet 1994

\title{
Photothermally modulated spatially resolved FMR detection of Walker modes in yttrium iron garnet spheres
}

\author{
O. von Geisau, F. Rödelsperger*, F. Schreiber, H. Benner* and J. Pelzl \\ Fakultät für Physik und Astronomie, AG Festkörperspektroskopie, Ruhr-Universität Bochum, \\ 44780 Bochum, Germany \\ * Institut für Festkörperphysik, Technische Hochschule Darmstadt, 64289 Darmstadt, Germany
}

\begin{abstract}
The photothermally modulated (PM) ferromagnetic resonance (FMR) combines the high sensitivity of conventional FMR and spatial resolution. This technique has been applied to a single-crystalline yttrium iron garnet (YIG) sphere with a diameter of $2 \mathrm{~mm}$. For the first time, the spatial variation of the high-frequency magnetization patterns known as Walker modes were visualized and it is thus shown that PM-FMR can even be applied to unfavourable geometries.
\end{abstract}

\section{INTRODUCTION}

Photothermal methods applied to the detection of ferromagnetic resonance (FMR) are able to furnish additional, spatially resolved information on magnetic excitations, not accessible to any other known technique [1-3]. Among the photothermal microwave detection schemes the photothermally modulated technique (PM-FMR) is of outstanding importance because of its high spatial resolution and its sensitivity being comparable to the one of conventional FMR measurements. In this work, PM imaging has been used to visualize, for the first time, the eigenmodes of the high-frequency magnetization in a sphere of yttrium iron garnet (YIG). These standing wave modes are commonly referred to as magnetostatic Walker modes [4]. Such experiments are interesting both from the technical and the physical point of view: on one hand, the results demonstrate the ability of this technique concerning the application to unfavourable geometries, on the other hand, the signal generation process for spheres is markedly different from the one for rectangular samples [3] as different spins precess with unequal relative phases.

\section{EXPERIMENTAL SETUP}

The experiments to be discussed in this paper were both performed with a broadband $(2-4 \mathrm{GHz})$ and a narrowband, resonant FMR spectrometer $(9.2 \mathrm{GHz})$. The sample, a $2 \mathrm{~mm}$ diameter single-crystalline YIG sphere, is placed in a magnetic field and, at the same time, is exposed to a continuous microwave and a modulated HeNe laser radiation, the latter being confined to a small spot. The PM-FMR signal is given by the oscillatory part of the conventional FMR diode signal which is measured in transmission (reflection) in the broadband (narrowband) experiment. Both techniques essentially yield the same results. The advantage of the resonant setup is its high sensitivity due to the high $Q$-factor of the cavity. But the use of a cavity also imposes severe restrictions on the applicability: The macroscopically large sample markedly disturbs the field distribution inside the cavity, thus not allowing for a correct impedance matching which is a prerequisite for resonant measurements. Using the nonresonant broadband setup the microwave excitation 

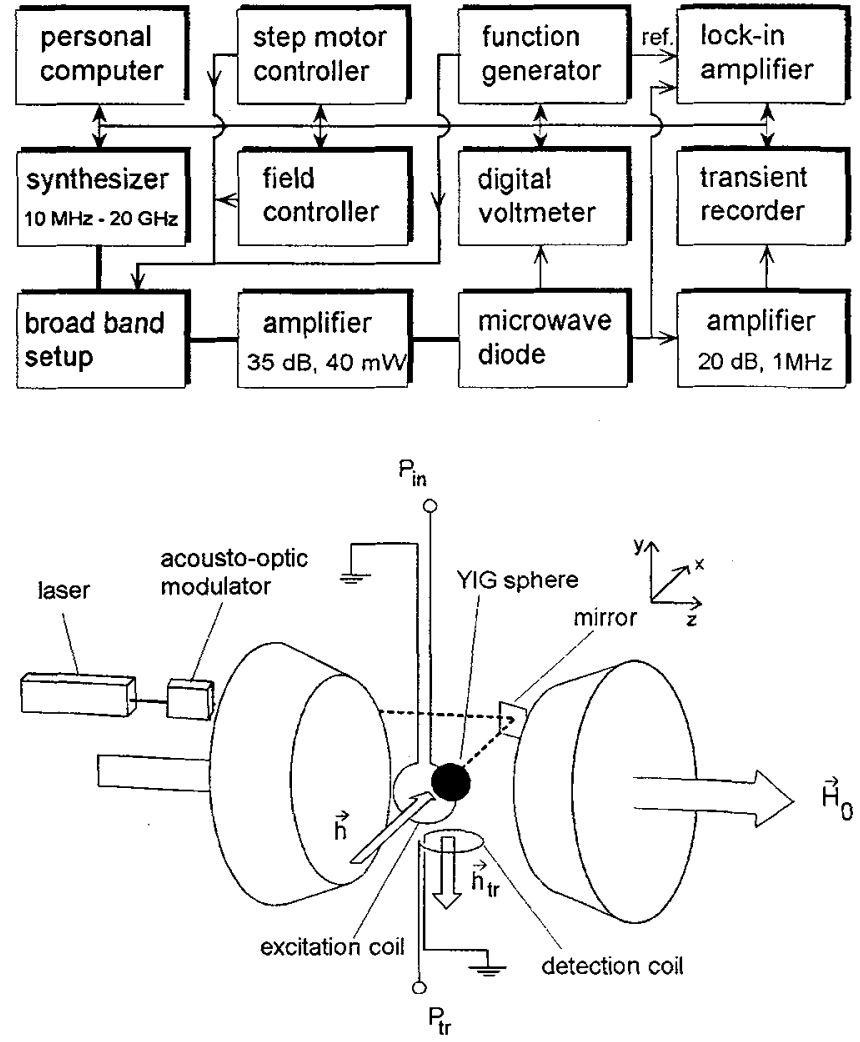

Fig. 1: Block diagram of the broadband FMR experiment for the investigation of Walker modes. The setup allows for modulated measurements as well as time resolved ones, the latter being of interest in the context of nonlinear dynamics.
Fig. 2: Setup of the broadband FMR experiment. The beam of a $12 \mathrm{~mW}$ HeNe laser is focussed onto the sample by a lens $(f=5 \mathrm{~cm})$ not shown in this sketch. The mirror is moved in two directions by stepper motors.

field at the sample location and with this the selection of the modes to be excited can be easily influenced. But the sensitivity as compared to the resonant technique is considerably lower.

A detailed description of the narrowband setup is given in [1]. The nonresonant setup is shown in Figs. 1 and 2. With this equipment the excitations of the high-frequency magnetization in the sample can be investigated in the frequency as well as in the time domain. The results presented in this paper have been recorded as a function of the modulation frequency only. Time resolved experiments are of interest as far as the nonlinear dynamics in the investigated systems is concerned [5]. Spatial resolution was achieved using a combination of a mirror movable into two perpendicular directions and a $f=5 \mathrm{~cm}$ lens (focal spot diameter $\approx 70 \mu \mathrm{m}$ ). In an area of $1 \mathrm{~mm}$ around the optical axis the beam displacement is proportional to the mirror rotation. For the imaging of Walker modes the PM-FMR signal was recorded on a quadratic grid. As only low order modes with moderate local variations of the absorbed microwave power were investigated, the loss of resolution towards the edges of this grid due to the non-perpendicular incidence of the laser beam is negligible. The microwave power in the resonant experiments $(Q \approx 1100)$ was chosen as $0.5 \mathrm{~mW}$, whereas in the nonresonant ones up to $100 \mathrm{~mW}$ had to be applied to the coil generating the microwave field. This difference is not only due to the $Q$ of the cavity but also reflects the fact that the coil was placed some $\mathrm{mm}$ away from the sample.

The sample heating due to the absorption of light causes changes in the saturation magnetization $M_{0}$ and therefore a frequency shift between -1 and $+2 \mathrm{MHz} / \mathrm{K}$ while the line width is 0.7 to $0.85 \mathrm{MHz}$ corresponding to 25 to $30 \mu \mathrm{T}$. The temperature rise in the sample was about $2 \mathrm{~K}$. The Walker modes were excited at fixed external magnetic field and the frequency of the synthesizer was adjusted using a PID-controller in a way that during the experiment one remained on a fixed point at half maximum of the resonance line.

The modulation frequencies used in the experiments were smaller than $300 \mathrm{~Hz}$ for the nonresonant experiments and could be as large as $10 \mathrm{kHz}$ for the narrowband setup. The step width used in the imaging 
experiments was varied between 22 and $50 \mu \mathrm{m}$, thus being at least four times smaller than the diffusion length in the sample $\left(\mu_{\mathrm{YIG}}(300 \mathrm{~Hz}) \approx 200 \mu \mathrm{m}\right)$.

\section{RESULTS AND DISCUSSION}

Fig. 3 shows the PM-image of the (210) Walker mode recorded at a microwave frequency of $2.8849 \mathrm{GHz}$ and a magnetic induction of $127.000 \mathrm{mT}$. The indices 2,1 and 0 describe the spatial pattern of the hfmagnetization $\mathbf{m}(\mathbf{r})$ [6]. The resonance frequency $\omega_{210}$ of this mode expressed in magnetic field units is obtained by solving the spherical electro-magnetic boundary value problem [7]:

$$
\frac{\omega_{210}}{\mu_{0} \gamma}=\underbrace{-\frac{1}{3} M_{0}}_{H_{\text {demstat }}}+\underbrace{\frac{1}{5} M_{0}}_{H_{\text {demam }}}+H_{0}
$$

In the above expression $\mu_{0}$ is the permeability of the vacuum, $\gamma$ the gyromagnetic ratio, $M_{0}$ the saturation magnetization and $H_{0}$ is the external magnetic field. Neglecting anisotropy fields, the field $\omega_{210} / \mu_{0} \gamma$ is composed of the external magnetic field $H_{0}$, a static demagnetizing contribution $H_{\text {dem,stat }}$ and a dynamic demagnetizing field $H_{d e m, d y n}$ which is connected with the distribution of the hf-magnetization $\mathbf{m}$ in the sample. Therefore, when locally heating the sample, the changes in $H_{d e m, s t a t}$ do not depend on the location

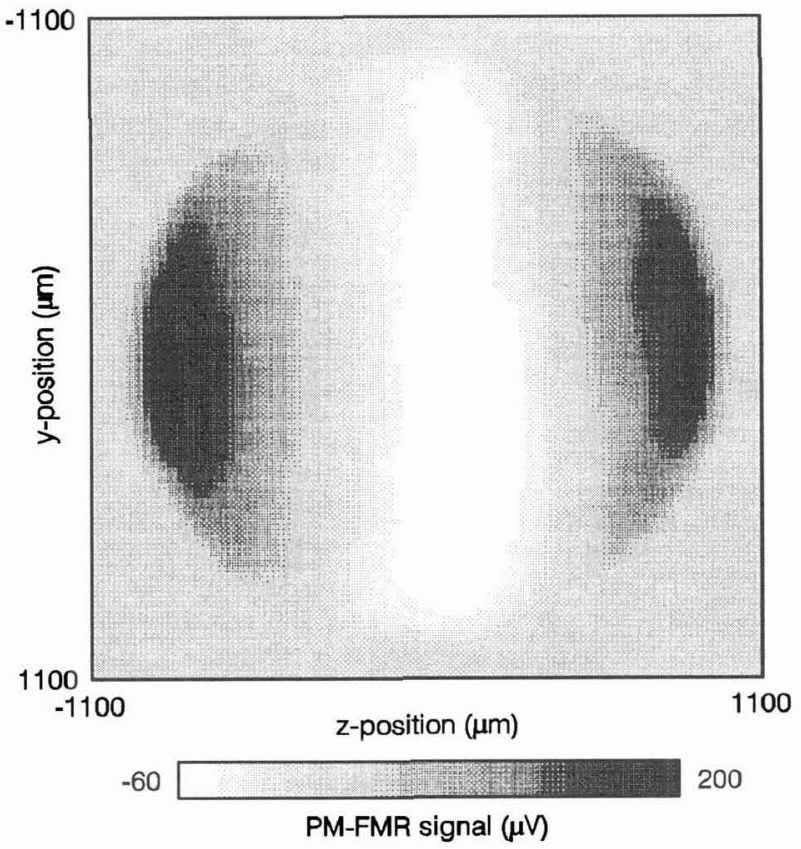

Fig. 3. PM-FMR image of the (210)-mode in a $2 \mathrm{~mm}$ YIG sphere. The experimental parameters are: Microwave frequency $=2.8849 \mathrm{GHz}$, microwave power $=10 \mathrm{dBm}$, magnetic field $=127.00 \mathrm{mT}$, modulation frequency $=270 \mathrm{~Hz}$. The PMsignal was recorded at a frequency higher than the resonance frequency.
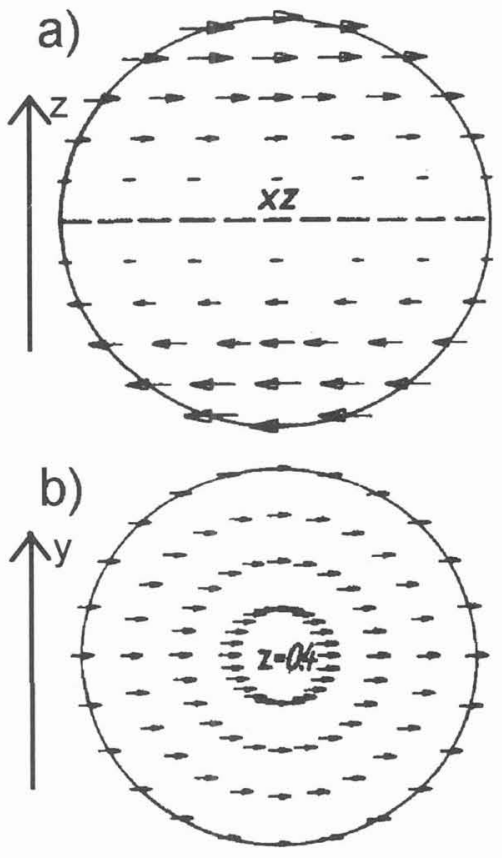

Fig. 4. Calculated distribution of the hfmagnetization $\mathbf{m}$ of the (210)-mode. The dashed line is a nodal line. The vectors precess around $z$. a) $x z$-plane, $y=0$. b) $x y$-plane, $z=0.4$ radius. (from [6]) 
of the laser focus. $H_{d e m, d y n}$, however, experiences the biggest changes where $\mathbf{m}$ is largest. In areas with non-vanishing $\mathbf{m}$ the first and the second term compete as they have opposite temperature coefficients $\partial$ $\omega_{210} / \partial T$. At a node of $\mathbf{m}$ the resonance line shifts to higher frequencies because of the positive temperature coefficient. Scanning the resonance line from low to high frequencies using a modulated illumination together with a $\mathrm{N}$-type crystal diode one obtains a derivative spectrum with negative values above the resonance frequency. In Fig. 3 this can be seen at $z, y=0$. Moving the laser beam at constant $y=0$ toward larger $z$-values one observes a change of the sign of the PM signal. This is due to the contribution of the dynamical demagnetizing field. The value of $H_{d e m, d y n}$ is only an average value. Thus, at locations with maximum $\mathbf{m}$, the line shift in negative direction can overcompensated. At fixed $z$ there is nearly no variation of the PM-FMR signal along $y$. These spatial variations of the PM signal agree with the predictions obtained from the calculated magnetization patterns [6] along the $z$ - (Fig. 4a) and the $y$-direction (Fig. 4b). The observed slight deviations are attributed to the distorted spot size of the laser near the margins of the sample.

The frequency dependent behaviour of the Walker modes was investigated with the narrowband setup. For modulation frequencies $v_{\text {mod }}$ above $100 \mathrm{~Hz}$ a $v_{\text {mod }}{ }^{-1}$ behaviour of the PM amplitude was observed as expected for a thermally thick plane sample [3]. For lower frequencies, the spherical geometry of the sample has to be taken into account. For that reason no $v_{m o d}{ }^{-1 / 2}$ dependence was found. In the region of the $v_{\text {mod }}{ }^{-1}$ decline of the PM amplitude the PM signal phase is expected to assume a value of $-90^{\circ}$. In the experiment, the PM signal phase approaches this value at $1 \mathrm{kHz}$, thus showing the influence of the spherical geometry at low modulation frequencies. The comparison of the frequency dependence of the amplitude and the phase indicates that the phase depends more sensitively on the geometry than the amplitude.

\section{CONCLUSION}

The PM-FMR technique was applied to the detection of Walker modes in spheres. The spatial distribution of the high-frequency magnetization of these magnetostatic eigenmodes has not been investigated before. The signal generation process was discussed and the findings were confirmed by the comparison with theoretical calculations of the spatial mode patterns.

\section{Acknowledgements}

This work is supported by the Deutsche Forschungsgemeinschaft, Kennwort "Photothermik".

\section{References}

[1] J. Pelzl and O. von Geisau, in Non-Destructive Evaluation, A. Mandelis, Ed., (Prentice Hall, Englewood Cliffs, 1994), pp. 237-320.

[2] O. von Geisau and J. Pelzl, in High Frequency Processes in Magnetic Materials, G. Srinivasan and A. N. Slavin, Eds., (World Scientific, River Edge NJ, 1994, in press)

[3] O. von Geisau, Photothermische Untersuchungen der FMR langwelliger kollektiver magnetischer Anregungen in YIG, PhD-Thesis, Ruhr-Universität Bochum, 1993

[4] L. R. Walker, Phys. Rev. 105 (1957) 390 - 399

[5] G. Wiese and H. Benner, Z. Phys. B - Condensed Matter 79 (1990) $119-131$

[6] P. Röschmann and H. Dötsch, Phys. Stat. Sol. B 82 (1977) $11-57$

[7] P. C. Fletcher and R. O. Bell, J. Appl. Phys. 30 (1959) 687 - 698 